Communications in Physics, Vol.23, No. 1 (2013), pp. 85-89

\title{
INFLUENCE OF THE FREQUENCY-CHIRP GAUSSIAN PULSE ON THE PULSE BROADENING IN THE DISPERSIVE FIBERS
}

\author{
BUI XUAN KIEN \\ Electric Power University \\ TRAN HAI HUNG \\ Nghe An Pedagogical College \\ TRINH DINH CHIEN \\ University of Science, Vietnam National University, Hanoi, Vietnam
}

\begin{abstract}
A signal optical pulse propagating in the fiber is affected by many factors, one of the most important among them is dispersion. Under the dispersive influence, the optical pulse is reshaped, particulally, its duration is changed. Especially, the pulse duration change of the optical short pulse with the frequency-chirp is unregular. It influences to the propagating distance in the fiber optic network.

In this paper, the expression of the signal optical pulse with the frequency-chirp propagated through the dispersive fiber is derived. This expression described the broadening of the pulse in propagating process (broadening factor), which depends on the frequence chirp and dispersive parameters. The influence of those parameters on the pulse broadening is simulated and discussed.
\end{abstract}

\section{INTRODUCTION}

As well known, in the optical communication, the most interesting problems are the pulse sharping and propagating distance. It is fact that the signal optical pulse propagating in the dispersive fiber is always affected by the nonlinear effects as disspersion, self-phase modulation and cross-phase modulation,...[1-5], which broadens (or narrowes) the pulse, expecially, the pulse with frequency-chirp and consequently, reduces the bit rate.

Up to now, the dispersive questions are interested in many works $[2,3]$, but till not paied attentions on the influence of the pulse with the frequency-chirp on the propagating distance in the fibers.

We present the expressions of the optical pulse propagated through the optical fiber and of the broadening factor in Sec. 2. The influence of the chirp and dispersive parameters on the broadening is simulated and discussed in Sec. 3.

\section{THE BROADENING FACTOR OF THE OPTICAL GAUSSIAN CHIRP PULSE IN THE DISPERSIVE FIBERS}

We consider a optical Gaussian pulse propages in the unreducing linear dispersive fiber. To describe the wave propagation of the picosecond pulse in the linear dispersive 
medium, the general Schrodinger equation [1] is reduced to:

$$
i \frac{\partial A}{\partial z}=+\frac{\beta_{2}}{2} \frac{\partial^{2} A}{\partial T^{2}}
$$

where $A$ is the slowly varying amplitude of the pulse envelope; $T=t-\frac{z}{v_{g}}$ is the time measured in the frame of the reference moving with the pulse at the group velocity $\mathrm{v}_{g} \beta_{2}=$ $\left(\frac{d^{2} \beta(\omega)}{d \omega^{2}}\right)_{\omega=\omega_{0}}$ is the coefficient described the group velocity dispersion, $\beta(\omega)$ is the wave propagation coefficient at frequency, $\omega_{0}$ is the central frequency.

For simplicity, the normalized time and amplitude are defined as following:

$$
\tau=\frac{T}{T_{0}} ; \quad U(z, \tau)=\frac{A}{\sqrt{P_{0}}}
$$

where $P_{0}, T_{0}$ is the amplitude peak and duration of the incident pulse, respectively.

Now, equation (1) is rewritten as:

$$
i \frac{\partial U}{\partial z}=\frac{\operatorname{sgn}\left(\beta_{2}\right)}{2 L_{D}} \frac{\partial^{2} U}{\partial \tau^{2}}
$$

where $\operatorname{sgn}\left(\beta_{2}\right)= \pm 1$ depending on the sign of $\beta_{2}$ (relating to dispersive character of the fiber) and $L_{D}=\frac{T_{0}^{2}}{\left|\beta_{2}\right|}$ is defined as the dispersive length.

As above consideration, the fiber is linear dispersive, so the equation (2) can be rewritten as

$$
i \frac{\partial U}{\partial z}=\frac{\beta_{2}}{2} \frac{\partial^{2} U}{\partial T^{2}}
$$

Equation (3) is solved by using the Fourier-transform method, such that

$$
U(z, T)=\frac{1}{2 \pi} \int_{-\infty}^{\infty} \tilde{U}(z, \omega) \cdot \exp (-i \omega T) d \omega=\frac{1}{2 \pi} \int_{-\infty}^{\infty} \tilde{U}(0, \omega) \cdot \exp \left(\frac{i \beta_{2} \omega^{2} z}{2}-i \omega T\right) d \omega
$$

With

$$
\tilde{U}(0, \omega)=\int_{-\infty}^{\infty} U(0, T) \cdot \exp (i \omega T) d T
$$

is the Fourier transform of function $U(z, T)$.

We are interesting in the chirped Gaussian pulse, for which the incident field is of the form as follow:

$$
U(0, T)=\exp \left[-\frac{(1+i C)}{2} \frac{T^{2}}{T_{0}^{2}}\right]
$$

where $\mathrm{C}$ is defined as a parameter described the frequency-chirp (i.e. the instantanneous frequency increasing linearly from the leading to the tailing edge) of the pulse [1].

Substituting (6) to (5) and consequence to (4), after integration we have:

$$
U(z, T)=\left(\frac{T_{0}}{\left[T_{0}^{2}-i \beta_{2} z(1+i C)\right]^{1 / 2}}\right) \exp \left[-\frac{(1+i C) T^{2}}{2\left[T_{0}^{2}-i \beta_{2} z(1+i C)\right]}\right]
$$


This expression shows that the chirp Gaussian pulse maintains its Gaussian shape on propagation, but its duration $T_{1}$ after propagating a distance $z$ is related to the initial duration $T_{0}$ by the relation:

$$
\sigma=\frac{T_{1}}{T_{0}}=\left[\left(1+\frac{C \beta_{2} z}{T_{0}^{2}}\right)^{2}+\left(\frac{\beta_{2} z}{T_{0}^{2}}\right)^{2}\right]^{1 / 2}
$$

The ratio $\sigma$ is defined as the broadening factor at distance $z$, which depends on the chirp $C$ and dispersive parameters $\beta_{2}$.

\section{THE INFLUENCE OF THE CHIRP AND DISPERSIVE PARAMETERS ON THE PULSE BROADENING}

To confirm that, the broadening of the Gaussian pulse with duration $T_{0}=100 \mathrm{ps}$ and different value of the chirp parameter, which propagates through the in different dispersvie fiber with length $z=100 \mathrm{~km}$ and dispersive parameter $\beta_{2}=-50,-20,20,50 \mathrm{ps}^{2} / \mathrm{km}$ [3] are simulated and illustrated in Fig. 1.

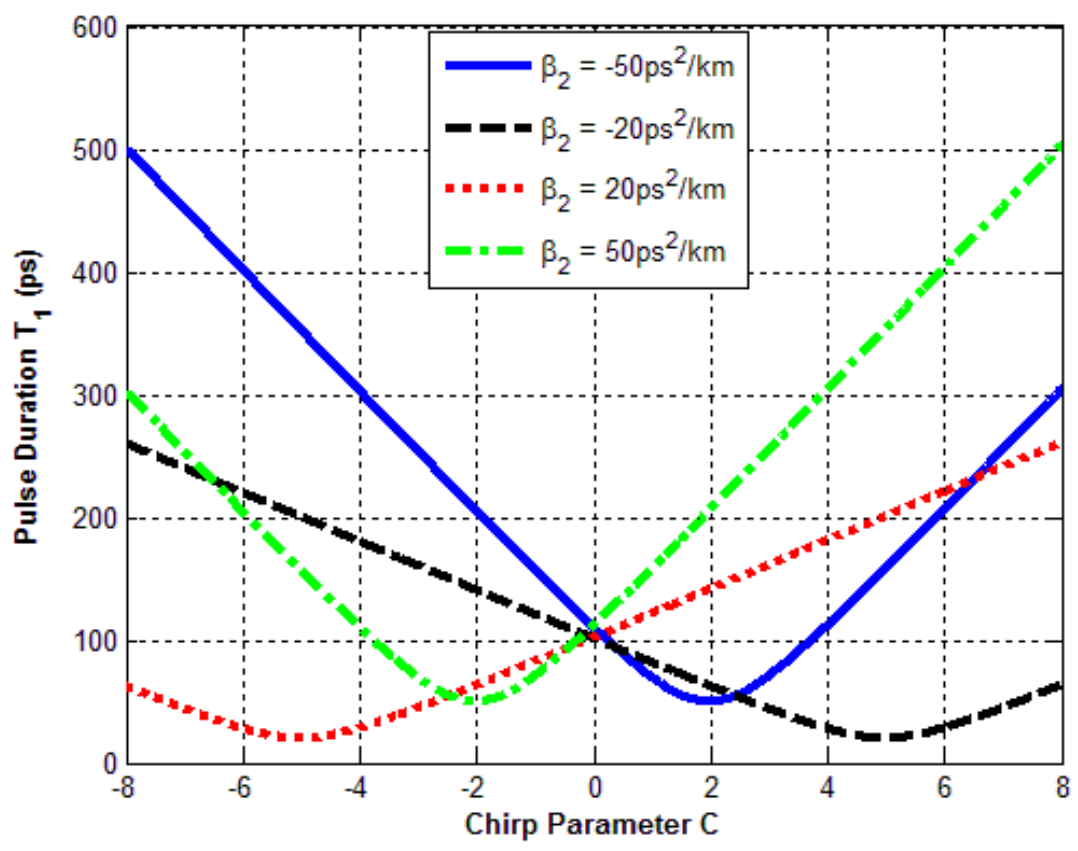

Fig. 1. Ouput pulse duration vs chirp parameter for fiber with length $100 \mathrm{~km}$ and dispersive parameters of: $-50 \quad \mathrm{ps}^{2} / \mathrm{km}$ (line);-20 $\mathrm{ps}^{2} / \mathrm{km}$ (dashs); $+20 \quad \mathrm{ps}^{2} / \mathrm{km}$ (dots)and $+50 \mathrm{ps}^{2} / \mathrm{km}$ (dashs-dots).

From Fig. 1 we can see that depending on the sign of parameters the pulse will be broadened or narrowed. In the normalous-dispersive $\left(\beta_{2}>0\right)$ fiber, the up-chirp $(C>0)$ pulse will be broadened, meanwhile the down-chirp $(C<0)$ pulse narrowed. In 
the unnormalous-dispersive $\left(\beta_{2}<0\right)$ fiber those processes are opposite. There is a value region of chirp and dispersive parameters, for which the shortening process is prominent than the broadening one and consequence, the minimum duration of the pulse appears. For the pulse without frequency chirp $(C=0)$, its duration always broadens because of the group velocity dispersion, however, the broadening factor is small $(\sigma=1.01 \div 1.12)$ for fiber with dispersion parameter changes in the large region from $10 \mathrm{ps}^{2} / \mathrm{km}$ to $50 \mathrm{ps}^{2} / \mathrm{km}$ (see Fig. 2). Moreover, there are two values of the chirp parameter, for which the broadening factor is equal to $1(\sigma=1)$, i.e. the duration of the output pulse is equal to that of the incident pulse. Two values of the chirp parameter changes in the different fibers. This leads to choice an optimal collection of the parameters, for which the pulse remains its shape after propagating through the given fiber. This phenomenon can be explained by the compensation of frequency chirp effect with dispersive one if the optimal parameter collection is chosen.

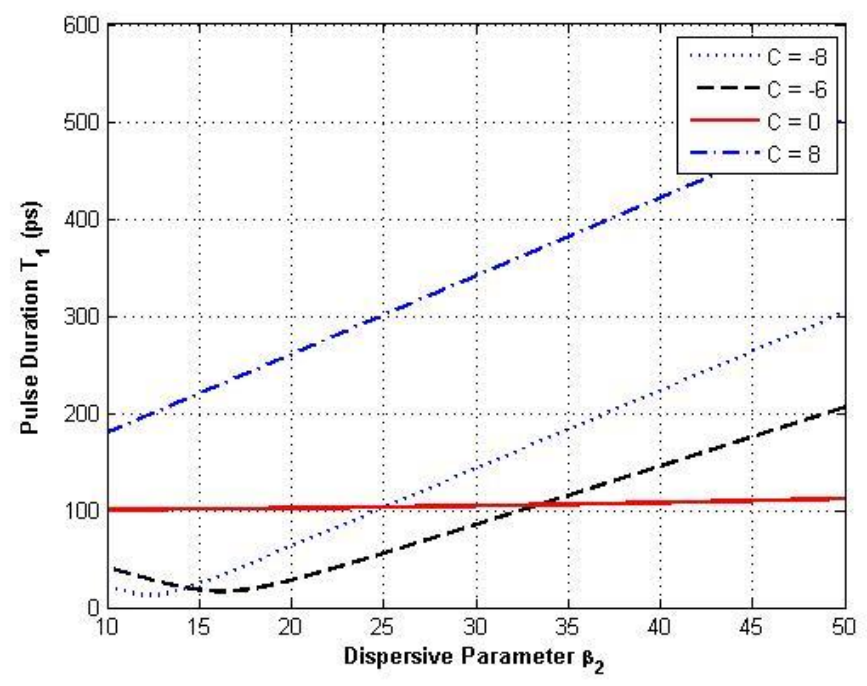

Fig. 2. Duration of the ouput pulse vs dispersive parameter for incident pulse with chirp.

In the fact, the communication fiber is the normalous dispersive medium, which has possitive dispersive parameter $\left(\beta_{2} i 0\right)$. So the pulse with up-chirp is always broadened when propagates in the fiber (dash-dot curve in Fig. 2). But, for the incident pulse with down-chirp, there is the compressing process in the fiber with dispersive parameter lies in certain interval. For example, if $\mathrm{C}=-8$, the pulse will be compressed in fiber with the dispersive parameter to $25 \mathrm{ps}^{2} / \mathrm{km}$ (dot curve in Fig.2 ). Meanwhile, if $\mathrm{C}=-6$, the pulse is compressed in the fiber with the dispersive parameter to $34 \mathrm{ps}^{2} / \mathrm{km}$ (dash curve in Fig. 2). All of the above comments means there is a collection of parameters, for that the propagated pulse is same as the incident one. For example, in Fig. 2, the collection of paramaters is: $T_{0}=100 \mathrm{ps}, L=100 \mathrm{~km}, \beta_{2}=25 \mathrm{ps}^{2} / \mathrm{km}$ and $C=-8$ or $T_{0}=100 \mathrm{ps}$, $L=100 \mathrm{~km}, \beta_{2}=34 \mathrm{ps}^{2} / \mathrm{km}$ and $C=-6$. 


\section{CONCLUSION}

From results we can affirm that: i) In the dispersive fiber, the duration of the optical pulse without frequency chirp is always changed; ii) The changing magnitude of the duration of the optical pulse (relating to broadening or narrowing) depends on the its chirp parameter and the dispersive parameter of the fiber; iii) There is a collection of parameters $\left(T_{0}, \beta_{2}, C, L\right.$ : for example, first collection: $T_{0}=100 \mathrm{ps}, \beta_{2}=25 \mathrm{ps}^{2} / \mathrm{km}$, $C=-8$ and $L=100 \mathrm{~km}$, and second collection: $T_{0}=100 \mathrm{ps}, \beta_{2}=34 \mathrm{ps}^{2} / \mathrm{km}, C=-6$ and $L=100 \mathrm{~km}$ ), for which the propagated chirp pulse did not change, i.e. the broadening factor $\sigma=1$.

\section{REFERENCES}

[1] G. P. Agrawal, Nonlinear Fiber Optics, fourth edition, pp. 50-58, Academic Press (2007).

[2] M. Stefanovi, P. Spalevic, D. Martinovic, M.Petrovic, Optical Communications 27 (2006) 305-309.

[3] A. Rostami and A. Andalib, Progress In Electromagnetics Research 75 (2007) 209-224.

[4] C. Rulliere, Femtosecond Laser Pulses, pp. 30-33, Springer (1998).

[5] D. Yongkui et al. Optical Communications 225 (2003) 363-369. 